\title{
Numerical simulation of carrier collection and recombination at grain boundaries in $\mathrm{Cu}(\mathrm{In}, \mathrm{Ga}) \mathrm{Se}_{2}$ solar cells \\ K. Taretto, and U. Rau
}

Citation: Journal of Applied Physics 103, 094523 (2008);

View online: https://doi.org/10.1063/1.2917293

View Table of Contents: http://aip.scitation.org/toc/jap/103/9

Published by the American Institute of Physics

\section{Articles you may be interested in}

Grain-boundary recombination in $\mathrm{Cu}(\mathrm{In}, \mathrm{Ga}) \mathrm{Se}_{2}$ solar cells

Journal of Applied Physics 98, 113704 (2005); 10.1063/1.2133906

The impact of charged grain boundaries on thin-film solar cells and characterization

Journal of Applied Physics 98, 063701 (2005); 10.1063/1.2042530

Detailed Balance Limit of Efficiency of $p$-n Junction Solar Cells

Journal of Applied Physics 32, 510 (1961); 10.1063/1.1736034

Local built-in potential on grain boundary of $\mathrm{Cu}(\mathrm{In}, \mathrm{Ga}) \mathrm{Se}_{2}$ thin films

Applied Physics Letters 84, 3477 (2004); 10.1063/1.1737796

Physics of grain boundaries in polycrystalline photovoltaic semiconductors

Journal of Applied Physics 117, 112807 (2015); 10.1063/1.4913833

Does the local built-in potential on grain boundaries of $\mathrm{Cu}(\mathrm{In}, \mathrm{Ga}) \mathrm{Se}_{2}$ thin films benefit photovoltaic performance of the device?

Applied Physics Letters 85, 2625 (2004); 10.1063/1.1793346

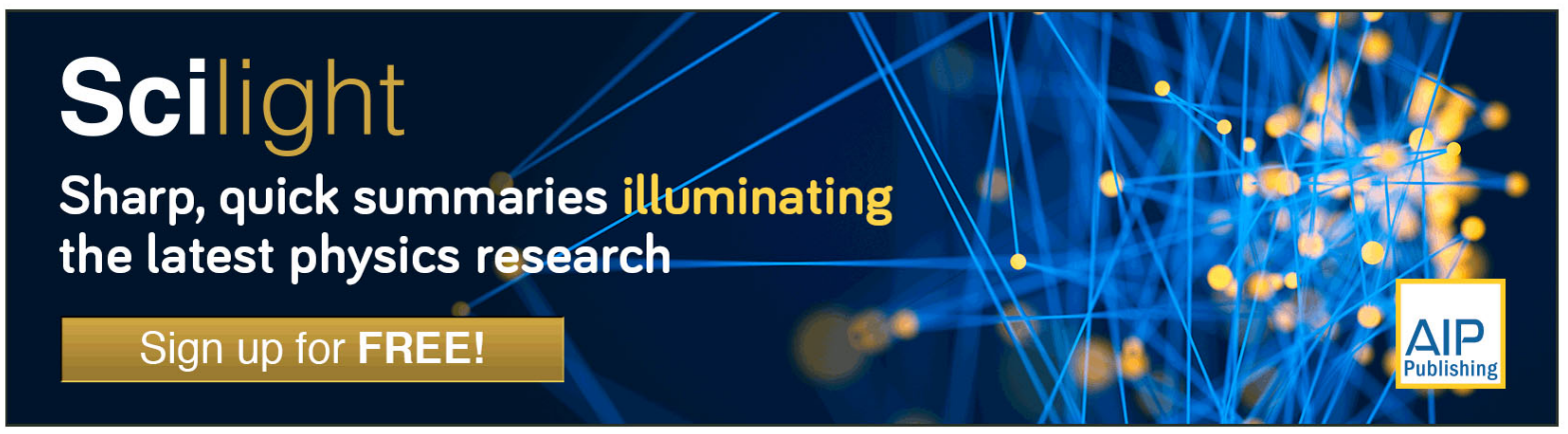




\title{
Numerical simulation of carrier collection and recombination at grain boundaries in $\mathrm{Cu}(\mathrm{In}, \mathrm{Ga}) \mathrm{Se}_{2}$ solar cells
}

\author{
K. Taretto ${ }^{1}$ and U. Rau ${ }^{2, a)}$ \\ ${ }^{1}$ Departamento de Electrotecnia, Universidad Nacional del Comahue, Buenos Aires 1400, 8300 Neuquén, \\ Argentina \\ ${ }^{2}$ IEF5-Photovoltaik, Forschungszentrum Jülich, 52425 Juelich, Germany
}

(Received 8 February 2008; accepted 2 March 2008; published online 14 May 2008)

\begin{abstract}
Two-dimensional numerical device simulations investigate the influence of grain boundaries (GBs) on the performance of $\mathrm{Cu}(\mathrm{In}, \mathrm{Ga}) \mathrm{Se}_{2}$ solar cells. We find that the electronic activity of grain boundaries can reduce the efficiency of $\mathrm{Cu}(\mathrm{In}, \mathrm{Ga}) \mathrm{Se}_{2}$ solar cells from $20 \%$ to below $12 \%$ making proper passivation of GBs a primary requirement for high efficiency. Cell efficiencies larger than $19 \%$ require GB defect densities below $10^{11} \mathrm{~cm}^{-2}$. Also, an internal band offset in the valence band due to a $\mathrm{Cu}$-poor region adjacent to the GBs could effectively passivate grain boundaries that are otherwise very recombination active. It is shown that such a barrier must be more than $300 \mathrm{meV}$ high and at least $3 \mathrm{~nm}$ wide to virtually suppress all grain boundary recombination. Contrariwise, such a barrier represents an obstacle for hole transport reducing carrier collection across grain boundaries that are not perpendicular to the cell surface. We further find that inverted grain boundaries lead to an accumulation of the short circuit current along the grain boundary, which in certain situations enhances the total short circuit current. However, we do not find any beneficial effect of any type of grain boundaries on the overall cell efficiency. (C) 2008 American Institute of Physics. [DOI: 10.1063/1.2917293]
\end{abstract}

\section{INTRODUCTION}

$\mathrm{Cu}(\mathrm{In}, \mathrm{Ga}) \mathrm{Se}_{2}$ (CIGS) solar cells provide the highest power conversion efficiency of all thin-film photovoltaic devices. ${ }^{1}$ The grain size $g$ of these polycrystalline absorber films hardly exceeds the film thickness $d$ of typically $d$ $=1.5-2.5 \mu \mathrm{m}$. The electronic activity of grain boundaries (GBs) in such a situation could be much more critical than in multicrystalline Si solar cells with $g$ being of the order of $g=5-10 \mathrm{~mm}$. Interestingly, solar cells made from polycrystalline $\mathrm{CuInSe}_{2}$ and CIGS absorbers reach efficiencies above $14 \%$ and $19 \%$ respectively, ${ }^{1,2}$ markedly outperforming their $12.5 \%$ efficient monocrystalline counterparts. ${ }^{3}$ This situation is very similar to that of polycrystalline CdTe solar cells but very different to that of multicrystalline/monocrystalline $\mathrm{Si}$ solar cells. During the past few years, considerable research effort was directed to understanding why the polycrystalline materials CdTe (Refs. 4 and 5) and CIGS (Refs. 6-17) yield such excellent photovoltaic absorbers. Especially, the question was discussed whether, and if yes, how GBs can be beneficial for CIGS and CdTe solar cells whereas they are detrimental for most other photovoltaic materials. In addition, there are several models that explain the low electronic activity of GBs in CIGS with the help of arguments concerning beneficial crystallography of $\mathrm{GBs}^{12}{ }^{12}$ extrinsic defect chemistry based on the beneficial effect of oxygen ${ }^{18,19}$ and sodium, ${ }^{20,21}$ as well as a self-passivation effect by an internal valence band offset. ${ }^{13,14}$

The present work uses two-dimensional numerical simulations of polycrystalline CIGS solar cells to investigate the influence of GBs on their electrical performance. The results

\footnotetext{
${ }^{\text {a) }}$ Author to whom correspondence should be addressed. Electronic mail: u.rau@fz-juelich.de.
}

presented here extend earlier simulations ${ }^{22,24}$ and incorporate analytical assessments of specific recombination and transport effects that may occur in polycrystalline CIGS. Concentrating on the question whether and under which circumstances CIGS cells may reach high efficiencies, the present simulations focus on the influence on solar cell performance of GB defect densities, bulk and GB defect energy and capture cross sections, grain sizes, and GB position (perpendicular and parallel to the cell surface). In order to obtain GB barrier heights of $100 \mathrm{meV}$ consistent with experimental data, ${ }^{6-9,12}$ our model requires a GB defect density of $N_{\text {it }}$ $\approx 2 \times 10^{12} \mathrm{~cm}^{-2}$. In such a situation, $19 \%$ efficiency cells are obtained only with a low GB electron capture cross section $\sigma_{\mathrm{it}, n}=10^{-18} \mathrm{~cm}^{2}$. Defects above midgap are shown to enhance the cell's short circuit current at defect densities above $2 \times 10^{12} \mathrm{~cm}^{-2}$, which ensure carrier population inversion around the GB. Additionally, we explore the possibilities for a polycrystalline semiconductor to outperform a single crystalline material in terms of photovoltaic performance. Our results show that this situation cannot be constructed by introducing GB defects into an absorber with excellent photovoltaic properties. Nevertheless, redistribution of a fixed amount of defects from the grain interior toward the GBs can be beneficial. Also, when starting with a relatively poor absorber bulk material, we find that additional GB defects may enhance at least the short circuit current.

It has been proposed that GBs in CIGS feature a hole barrier due to a $\mathrm{Cu}$-poor layer surrounding the $\mathrm{GB},{ }^{13,15}$ which would strongly reduce GB recombination. Here, we obtain an analytical expression that models the recombination rate at such a barrier by assuming different GB capture cross sections $\sigma_{\mathrm{it}, n}$ and $\sigma_{\mathrm{it}, p}$ for electrons and holes. In cells where the GB is perpendicular to the cell surface, the simu- 


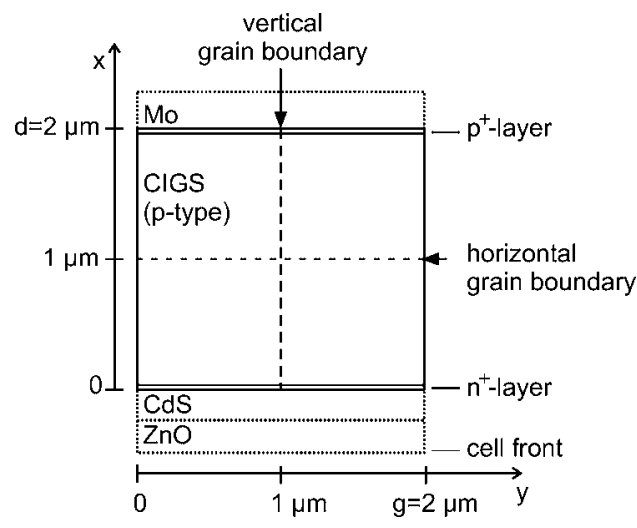

FIG. 1. Solar cell geometry considered in the simulations, with a cell thickness $d=2 \mu \mathrm{m}$ and a grain size $g=2 \mu \mathrm{m}$, with a vertical GB at the coordinate $y=1 \mu \mathrm{m}$ (used in Secs. III and IV) or a horizontal GB (Sec. V). At the front of the cell, a $\mathrm{ZnO}$ top contact is followed by a CdS layer which forms a junction with the CIGS absorber. Our model mimics this junction using a $50 \mathrm{~nm}$ thick $n^{+}$-type CIGS layer placed at $x=0$. The Mo back contact and its corresponding $\mathrm{MoSe}_{2}$ layer (not shown) are modeled by a $50 \mathrm{~nm}$ thick $p^{+}$-CIGS layer. The dotted layers are included in the calculations of the optical generation rate only.

lations show that a significant improvement of the efficiency requires an internal valence band offset $\Delta E_{V}$ $=k T \log \left(\sigma_{\mathrm{it}, n} / \sigma_{\mathrm{it}, p}\right)>300 \mathrm{meV}$, where $k T$ denotes the thermal energy. Up until this point, our results are consistent with earlier work. ${ }^{22-24}$ Here, we also show that the width of the region with lowered valence band energy must be larger than $3 \mathrm{~nm}$, otherwise quantum-mechanical tunneling reduces the passivation effect. If the GB is parallel to the cell surface, the high barriers represent an obstacle for hole transport that strongly reduce the device efficiency.

This paper is organized as follows: In Sec. II, we introduce the model, detailing the geometry, parameters, and solving method utilized for the simulations. Section III presents the simulation results for the case of a vertical grain boundary, followed by the analysis of the effects of the GB hole barrier in Sec. IV. Finally, Sec. V presents the simulations for horizontal GBs and in Sec. VI, we summarize and discuss the results.

\section{MODEL}

Figure 1 displays the assumed geometry of the $\mathrm{ZnO}$ / CdS/CIGS solar cell structure. For the computations discussed in Secs. III and IV, we assume a vertical GB placed along the center of the absorber yielding a columnar grain with a grain width of $g=2 \mu \mathrm{m}$. Section III C adds results with a fourfold smaller grain size of $g=0.5 \mu \mathrm{m}$. In Sec. V, we assume a horizontal GB dividing the absorber into two equal parts, as shown by the dashed line in Fig. 1. The CIGS absorber of thickness $d=2 \mu \mathrm{m}$ has a band gap energy $E_{g}$ $=1.15 \mathrm{eV}$. The photogeneration profile $G(x)$ is calculated from a coherent optical model ${ }^{25}$ for the complete $(300 \mathrm{~nm}) \mathrm{ZnO} /(50 \mathrm{~nm}) \mathrm{CdS} /(2 \mu \mathrm{m}) \mathrm{CIGS} / \mathrm{Mo}$ stack illuminated with a $100 \mathrm{~mW} / \mathrm{cm}^{2} \mathrm{AM} 1.5 \mathrm{G}$ spectrum. ${ }^{26}$ The generation profile inserted in our simulations corresponds to the absorption in the CIGS layer. The injection of carriers photogenerated in the CdS layer into the CIGS layer is incorporated by adding $30 \%$ of the CdS absorption ${ }^{27}$ into the CIGS

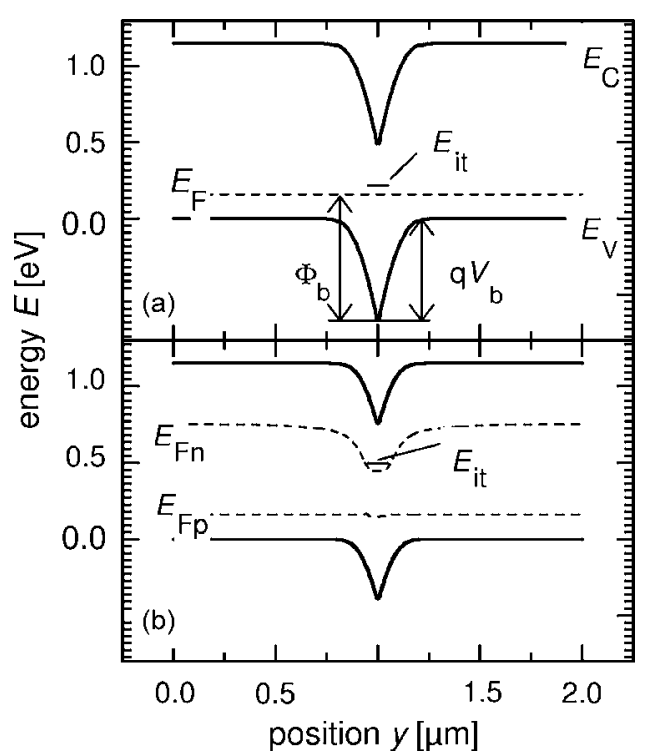

FIG. 2. Calculated band diagram in a CIGS solar cell under thermodynamic equilibrium (a) and during illumination under short circuit conditions (b), across a grain boundary (GB) located at $y=1 \mu \mathrm{m}$ (see Fig. 1). The energies $E_{V}$ and $E_{C}$ are the energies of the valence and conduction band edge, respectively, and $E_{\mathrm{it}}$ is the interface defect energy, here located at $0.880 \mathrm{eV}$ above the valence band edge. Additionally shown are the GB barrier height $\Phi_{b}$ and the band-bending energy $q V_{b}$. In part b, the hole and electron quasiFermi levels $E_{F p}$ and $E_{F n}$, are defined. The assumed GB trap density is $N_{\text {it }}$ $=4 \times 10^{12} \mathrm{~cm}^{-2}$.

generation rate profile at coordinate $x=0$, i.e., at the $\mathrm{CdS} /$ CIGS interface. The integral of the resulting $G(x)$ profile yields a maximum available short circuit current density $J_{\mathrm{SC}}^{\max }=33.5 \mathrm{~mA} / \mathrm{cm}^{2}$.

If not explicitly stated otherwise, we use the following material and device parameters: effective densities of states $N_{C}=10^{18} \mathrm{~cm}^{-3}$ and $N_{V}=10^{19} \mathrm{~cm}^{-3}$ for the conduction and valence bands, acceptor density $N_{A}=2 \times 10^{16} \mathrm{~cm}^{-3}$, mobilities $\mu_{p}=10 \mathrm{~cm}^{2} \mathrm{~V} \mathrm{~s}^{-1}$ for holes and $\mu_{n}=100 \mathrm{~cm}^{2} \mathrm{~V} \mathrm{~s}^{-1}$ for electrons, and relative dielectric constant $\varepsilon=10$. We have assumed zero recombination at the CdS/CIGS interface as well as at the Mo back contact.

The electronic activity of GBs is modeled by defects situated at energies $E_{\mathrm{it}}-E_{V}=270,605$, or $880 \mathrm{meV}$, where $E_{V}$ denotes the energy of the valence band maximum. One-half of the defects are assumed to be donorlike, the other half acceptorlike such that, in the limit of a high interface defect concentration $N_{\mathrm{it}}$, the Fermi energy $E_{F}$ is pinned at $E_{\mathrm{it}}$. A pinning position at $E_{\mathrm{it}}-E_{V}=270 \mathrm{meV}$ would lead to a band bending of about $100 \mathrm{meV}$ at the GB that is compatible with the available experimental data ${ }^{6-9,12}$ on CIGS, $E_{\mathrm{it}}-E_{V}$ $=880 \mathrm{meV}$ leads to type inversion at the grain boundary if $N_{\text {it }}$ is sufficiently high, whereas $E_{\mathrm{it}}-E_{V}=605 \mathrm{meV}$ corresponds to a trap level at the intrinsic Fermi energy $E_{i}$, i.e., a worst case scenario at equal capture cross sections for electrons and holes for the GB defects. Specific parameters that are used for the calculations presented in Figs. 2-8, 10, 11, 13, and 14 are summarized in Table I.

The simulation of the electronic transport is carried out for the CIGS part of the device only, by solving the stationary Poisson equation, and the current continuity equations combining drift and diffusion currents. ${ }^{28}$ In the continuity 


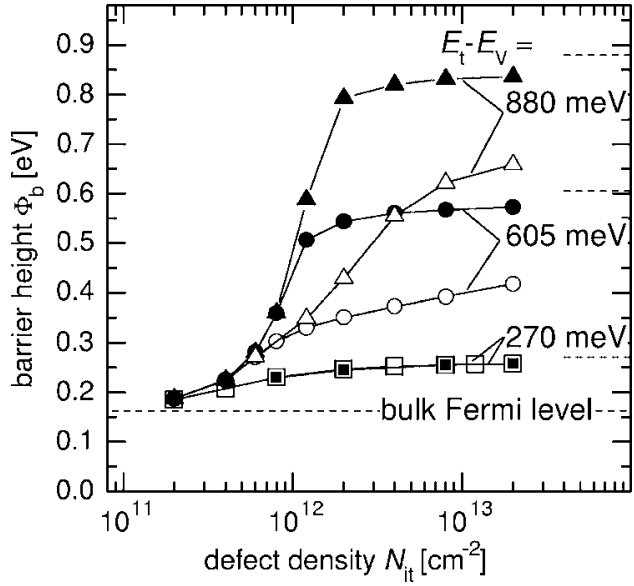

FIG. 3. Grain boundary barrier heights $\Phi_{b}$ with increasing GB defect density $N_{\text {it }}$ calculated in CIGS solar cells at a depth of $x=1 \mu \mathrm{m}$ (see Fig. 2) under illuminated, short circuit conditions (open symbols), and in the dark (full symbols). Shallow defect levels (squares) located at $E_{\mathrm{it}}-E_{V}$ $=270 \mathrm{meV}$ above the valence band edge yield small barrier heights that are only weakly increased by the addition of defect levels, and are unaffected by illumination. Defects at $E_{\mathrm{it}}-E_{V}=605 \mathrm{meV}$ (circles) and $E_{\mathrm{it}}-E_{V}$ $=880 \mathrm{meV}$ (triangles) build up significant GB barriers with increasing density $N_{\mathrm{it}}$, saturating at $N_{\mathrm{it}}=4 \times 10^{12} \mathrm{~cm}^{-2}$ in the dark due to Fermi level pinning. These high barriers are drastically reduced by illumination (open circles, open triangles).

equations, we insert the difference between the photogeneration profile $G(x)$ and the local net recombination rate, calculated as the sum of radiative and nonradiative defect-level recombination. The net radiative recombination rate is locally evaluated using the expression

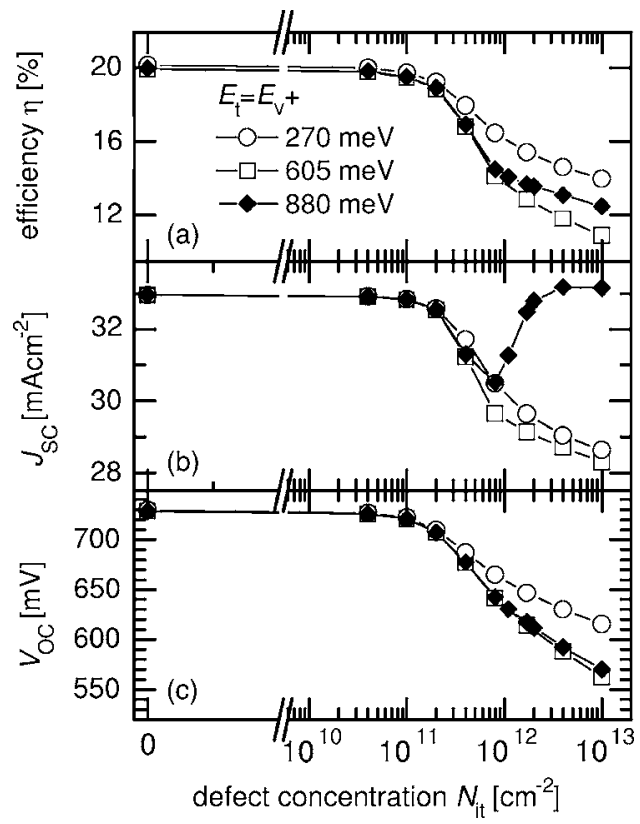

FIG. 4. Calculated efficiency $\eta$, short circuit current density $J_{\mathrm{SC}}$, and open circuit voltage $V_{\mathrm{OC}}$ for a CIGS solar cell with grain size $g=2 \mu \mathrm{m}$. The doping concentration is $N_{A}=2 \times 10^{16} \mathrm{~cm}^{-3}$ and the bulk defect concentration is $N_{\mathrm{bt}}=2 \times 10^{15} \mathrm{~cm}^{-3}$. Bulk as well as GB defect levels use equal electron and hole capture cross sections $\sigma_{\mathrm{bt}, n / p}=\sigma_{\mathrm{it}, n / p}=10^{-15} \mathrm{~cm}^{2}$ and energy levels at 270/605/880 $\mathrm{meV}$ (squares, circles, diamonds) above the valence band edge.

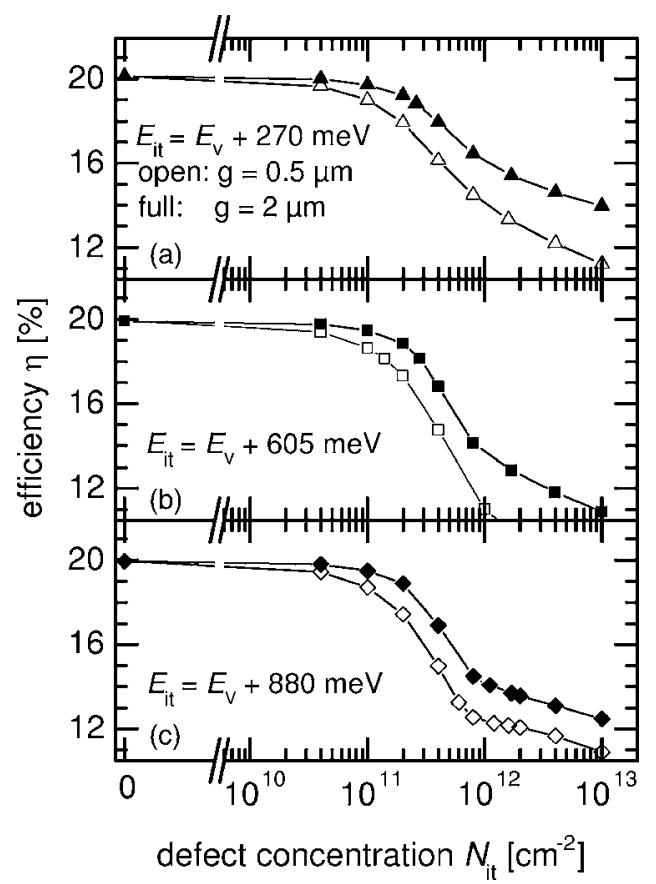

FIG. 5. Simulated dependence of cell efficiency $\eta$ on the defect concentration $N_{\text {it }}$ for grain sizes $g=0.5 \mu \mathrm{m}$ (open symbols) and $2 \mu \mathrm{m}$ (full symbols), and GB trap energies $270 \mathrm{meV}$ (a), $605 \mathrm{meV}$ (b), and $880 \mathrm{meV}$ (c) above the valence band edge. With the assumed capture cross sections of $\sigma_{\mathrm{bt}, n / p}$ $=\sigma_{\mathrm{it}, n / p}=10^{-15} \mathrm{~cm}^{-2}$, efficiencies over $19 \%$ are reached by cells with defect concentrations below $N_{\mathrm{it}}=2 \times 10^{11} \mathrm{~cm}^{-2}(g=2 \mu \mathrm{m})$ or $N_{\mathrm{it}}=10^{11} \mathrm{~cm}^{-2}(g$ $=0.5 \mu \mathrm{m})$.

$$
R_{\mathrm{rad}}=B_{\mathrm{rad}}\left(n p-n_{i}^{2}\right),
$$

where we assume $B_{\text {rad }}=6 \times 10^{-11} \mathrm{~cm}^{3} \mathrm{~s}^{-1}$, according to optical characterizations in CIGS thin films, ${ }^{29} n$ and $p$ are local electron and hole concentrations, respectively, and $n_{i}$ is the intrinsic carrier concentration. The bulk defect occupancy and recombination rate are evaluated using Shockley-ReadHall (SRH) statistics at the assumed monoenergetic donor/

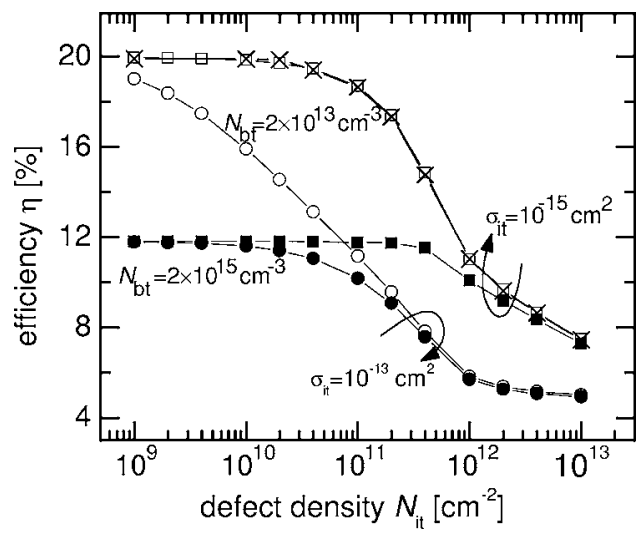

FIG. 6. Simulated dependence of cell efficiency $\eta$ on the defect concentration $N_{\text {it }}$ for grain sizes $g=0.5 \mu \mathrm{m}$ for bulk materials with defect concentrations $N_{\mathrm{bt}}=2 \times 10^{15} \mathrm{~cm}^{-3}$ (open symbols) and $N_{\mathrm{bt}}=2 \times 10^{13} \mathrm{~cm}^{-3}$ (full symbols) and a capture cross section $\sigma_{\mathrm{bt}, n / p}=10^{-13} \mathrm{~cm}^{2}$. The cell efficiencies decrease with increasing $N_{\text {it }}$ to levels that depend on the assumed capture cross sections $\sigma_{\mathrm{it}}=\sigma_{\mathrm{it}, n / p}=10^{-15} \mathrm{~cm}^{2}$ (full and open squares) and $\sigma_{\text {it }}$ $=10^{-13} \mathrm{~cm}^{2}$ (full and open circles) at the GBs. The data for a deeper bulk defect $E_{\mathrm{bt}}-E_{V}=605 \mathrm{meV}$ (crosses) with $N_{\mathrm{bt}}=2 \times 10^{15} \mathrm{~cm}^{-3}$ and $\sigma_{\mathrm{bt}, n / p}$ $=10^{-15} \mathrm{~cm}^{2}$ [cf. Fig. 5(b)] show no difference to the data obtained for shallower bulk defects $E_{\mathrm{bt}}-E_{V}=350 \mathrm{meV}$ (open squares). 


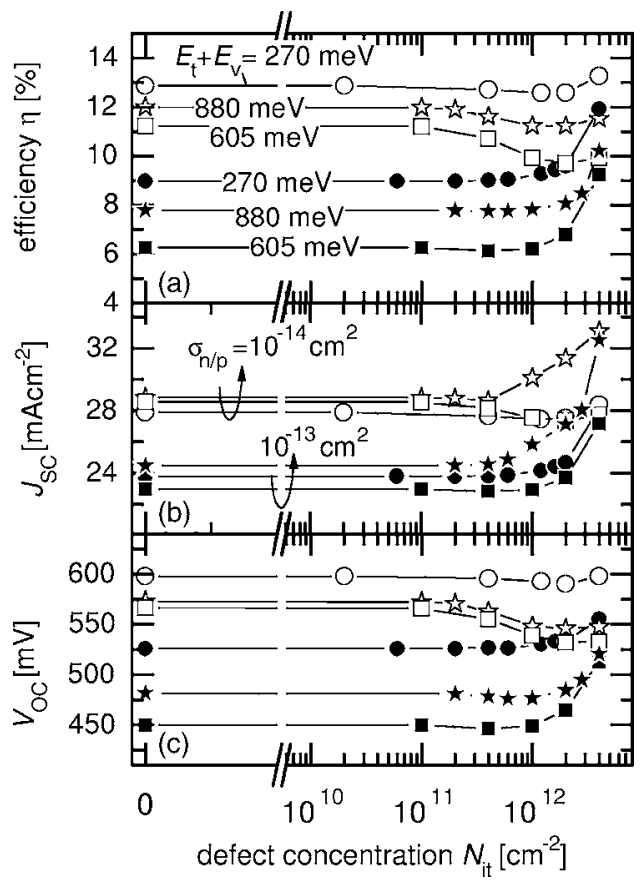

FIG. 7. Efficiency $\eta$, short circuit current density $J_{\mathrm{SC}}$, and open circuit voltage $V_{\mathrm{OC}}$ for an average defect density $N_{m}=2 \times 10^{16} \mathrm{~cm}^{-3}$. The increase in the concentration $N_{\text {it }}$ of interface defects implies a reduction of the concentration $N_{\mathrm{bt}}$ of bulk defects following $N_{\mathrm{bt}}=N_{m}-N_{\mathrm{it}} / g$. Open symbols correspond to data with capture cross sections $\sigma_{\mathrm{bt}, n / p}=\sigma_{\mathrm{it}, n / p}=10^{-14} \mathrm{~cm}^{2}$ while closed symbols use $\sigma_{\mathrm{bt}, n / p}=\sigma_{\mathrm{it}, n / p}=10^{-13} \mathrm{~cm}^{2}$.

acceptor defect level ${ }^{30}$ with a concentration $N_{b t}$. The grain boundary is modeled as a true internal surface, where we specify the charge density,

$$
Q_{\mathrm{it}}=\frac{q N_{\mathrm{it}}}{2}\left(1-2 f_{\mathrm{it}}\right),
$$

per unit area, where $q$ denotes the elementary charge and $f_{\text {it }}$ the occupation function of the assumed donor/acceptor GB, as established by SRH statistics, which also yield the GB surface recombination rate

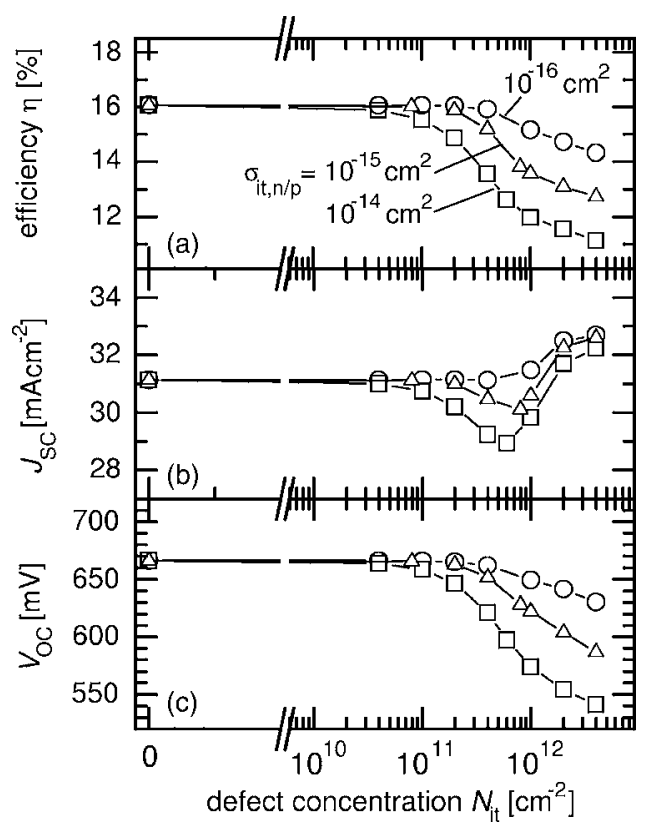

FIG. 8. Simulated efficiency $\eta$, short circuit current density $J_{\mathrm{SC}}$, and open circuit voltage $V_{\mathrm{OC}}$ as a function of the concentration $N_{\text {it }}$ of GB defects with energy $E_{\mathrm{it}}=E_{V}+880 \mathrm{meV}$ and capture cross sections $\sigma_{\mathrm{it}, p / n}$ $=10^{-14} / 10^{-15} / 10^{-16} \mathrm{~cm}^{2}$ (squares, triangles, circles). The bulk traps are placed at $605 \mathrm{meV}$ above valence band edge, and have a density $N_{\mathrm{bt}}=2$ $\times 10^{15} \mathrm{~cm}^{-3}$ and capture cross sections $\sigma_{\mathrm{bt}, p / n}=10^{-14} \mathrm{~cm}^{2}$.

$$
R_{\mathrm{it}}=\frac{N_{\mathrm{it}} v_{\mathrm{th}}\left(n_{\mathrm{it}} p_{\mathrm{it}}-n_{i}^{2}\right)}{\sigma_{\mathrm{it}, p}^{-1}\left(n_{\mathrm{it}}+n_{1, \mathrm{it}}\right)+\sigma_{\mathrm{it}, n}^{-1}\left(p_{\mathrm{it}}+p_{1, \mathrm{it}}\right)},
$$

where $v_{\text {th }}$ is the carrier thermal velocity, $N_{\text {it }}$ is the GB defect density per unit area (i.e., the sum of acceptor and donor states), $n_{\mathrm{it}}, p_{\mathrm{it}}$ are the local electron and hole concentrations at a given point of the GB line, and

$$
n_{1, \mathrm{it}}=N_{C} \exp \left(\frac{E_{\mathrm{it}}-E_{C}}{k T}\right),
$$

and

TABLE I. Defect energies $E_{\mathrm{bt}}$, defect concentrations $N_{\mathrm{bt}}$, and capture cross sections $\sigma_{\mathrm{bt}}$ for the bulk defects as well as the corresponding quantities $E_{\mathrm{it}}, N_{\mathrm{it}}$, and

\begin{tabular}{|c|c|c|c|c|c|c|c|}
\hline Figure & $\begin{array}{c}g \\
(\mu \mathrm{m})\end{array}$ & $\begin{array}{l}E_{\mathrm{bt}}-E_{V} \\
(\mathrm{meV})\end{array}$ & $\begin{array}{c}N_{\mathrm{bt}} \\
\left(\mathrm{cm}^{-3}\right)\end{array}$ & $\begin{array}{c}\sigma_{\mathrm{bt}} \\
\left(\mathrm{cm}^{2}\right)\end{array}$ & $\begin{array}{l}E_{\mathrm{it}}-E_{V} \\
(\mathrm{meV})\end{array}$ & $\begin{array}{c}N_{\mathrm{it}} \\
\left(\mathrm{cm}^{-2}\right)\end{array}$ & $\begin{array}{c}\sigma_{\mathrm{it}} \\
\left(\mathrm{cm}^{2}\right)\end{array}$ \\
\hline 2 & 2.0 & 880 & $2 \times 10^{15}$ & $10^{-15}$ & 880 & $4 \times 10^{12}$ & $10^{-15}$ \\
\hline 3 & 2.0 & $270,605,880$ & $2 \times 10^{15}$ & $10^{-15}$ & $270,605,880$ & $2 \times 10^{11}-2 \times 10^{13}$ & $10^{-15}$ \\
\hline 4 & 2.0 & $270,605,880$ & $2 \times 10^{15}$ & $10^{-15}$ & $270,605,880$ & $0-10^{13}$ & $10^{-15}$ \\
\hline 5 & $2.0 / 0.5$ & $270,605,880$ & $2 \times 10^{15}$ & $10^{-15}$ & $270,605,880$ & $0-10^{13}$ & $10^{-15}$ \\
\hline 6 & 0.5 & 350,605 & $2 \times 10^{13}, 2 \times 10^{15}$ & $10^{-13}$ & 605 & $10^{9}-10^{13}$ & $10^{13}, 10^{15}$ \\
\hline 7 & 2.0 & $270,605,880$ & $(0-2) \times 10^{16}$ & $10^{-13}, 10^{-14}$ & $270,605,880$ & $(0-4) \times 10^{12}$ & $10^{-13}, 10^{-14}$ \\
\hline 8 & 2.0 & 605 & $2 \times 10^{15}$ & $10^{-14}$ & 880 & $(0-4) \times 10^{12}$ & $10^{-16}-10^{-14}$ \\
\hline 9 & 2.0 & 605 & $2 \times 10^{15}$ & $10^{-14}$ & 880 & $8 \times 10^{10}-4 \times 10^{12}$ & $10^{-15}$ \\
\hline 11 & 2.0 & $270,605,880$ & $2 \times 10^{15}$ & $10^{-15}$ & $270,605,880$ & $4 \times 10^{12}$ & $\begin{array}{l}\text { Electrons: } 10^{-15} \\
\text { Holes: } 10^{-21}-10^{-15}\end{array}$ \\
\hline 12 & 2.0 & 270 & $2 \times 10^{15}$ & $10^{-15}$ & 270 & $4 \times 10^{11}, 4 \times 10^{12}$ & $\begin{array}{l}\text { Electrons: } 10^{-15} \\
\text { Holes: } 10^{-21}-10^{-15}\end{array}$ \\
\hline 14 & 2.0 & 270 & $2 \times 10^{15}$ & $10^{-15}$ & 270 & $4 \times 10^{11}, 4 \times 10^{12}$ & $\begin{array}{l}\text { Electrons: } 10^{-15} \\
\text { Holes: } 10^{-21}-10^{-15}\end{array}$ \\
\hline
\end{tabular}
$\sigma_{\text {it }}$ for the interface defects as used in the computations for Figs. 2-9, 11, 12, and 14. The sign "-" between two numbers indicates a covered range of the corresponding variable. 


$$
p_{1, \mathrm{it}}=N_{V} \exp \left(\frac{E_{V}-E_{\mathrm{it}}}{k T}\right),
$$

are auxiliary quantities. ${ }^{30}$

The two drift-diffusion equations together with the Poisson equation are simultaneously solved with a finite-element method using the software FEMLAB, ${ }^{31}$ which is set to solve all equations simultaneously. An iterative Newton scheme for highly nonlinear problems is run on a strongly irregularly spaced finite-element mesh consisting of about 5000 s-order rectangular elements. On the front and back surfaces, we use mixed boundary conditions. Majority carriers are set to a fixed concentration given by the equilibrium value, while for minority carriers we impose a zero-flux condition, i.e., zero surface recombination. The electrostatic potential is set to zero at the front surface, and at the back surface to the sum of the applied voltage and the internal built-in voltage that results from calculating the electrostatics in thermal equilibrium. The mesh spacing immediately around the GB is typically $2.5 \mathrm{~nm}$, while the minimum mesh spacing is $0.05 \mathrm{~nm}$ in the front space-charge region.

\section{VERTICAL GRAIN BOUNDARIES}

\section{A. Grain boundary barrier heights and type inversion}

For the electronic activity of GBs, we have to consider two different effects: first the areal charge density $Q_{\text {it }}$ accumulated at the GB defects as given by Eq. (2) induces a band bending in the bulk neighboring the GB interface. Figure 2(a) shows a calculated band diagram under equilibrium conditions where the Fermi energy $E_{F}$ at the GB is pinned to the energy $E_{\mathrm{it}}$ of the interface defects resulting in a band bending $q V_{b}$. The second consequence of GB defects results from their recombination activity. Under illumination, the quasiFermi levels $E_{F n}$ and $E_{F p}$ for electrons and holes are split, as shown in Fig. 2(b). The nonequilibrium charge carriers recombine following Eq. (3). In Eq. (3), the recombination current is not only proportional to the defect density $N_{\text {it }}$ but it depends via Eqs. (4) and (5) on the defect energy $E_{\mathrm{it}}$. Additionally, the band bending induced by the defect charge $Q_{\mathrm{it}}$ influences the free carrier concentrations $n, p$, and, consequently, also the recombination current. According to Eq. (3), a situation where $n=p$ is the most uncomfortable one at equal capture cross sections.

Figure 3 shows calculated barrier heights $\Phi_{b}$ across the GB, as defined in Fig. 2(a). For these calculations, we have assumed defects with capture cross sections $\sigma_{n / p}$ $=10^{-15} \mathrm{~cm}^{2}$ and energies $E_{\mathrm{it}}=E_{V}+270,605$, and $880 \mathrm{meV}$ with densities ranging from $N_{\text {it }}=2 \times 10^{11}$ to $2 \times 10^{13} \mathrm{~cm}^{-2}$. In addition to the doping density $N_{A}=2 \times 10^{16} \mathrm{~cm}^{-3}$, we consider a bulk defect density of $N_{\mathrm{bt}}=2 \times 10^{15} \mathrm{~cm}^{-3}$ that limits the lifetime of the minority carriers in the bulk of the material. Under equilibrium conditions (full symbols, in Fig. $3)$ and with low interface defect density $\left(N_{\text {it }}=2 \times 10^{11}\right)$, the barrier height $\Phi_{b}$ virtually equals the energy distance $E_{F}$ $-E_{C} \approx 160 \mathrm{meV}$ in the bulk for all defect energies $E_{\mathrm{it}}$, i.e., there is no energy barrier at the GB interface. Within the range from $N_{\mathrm{it}}=8 \times 10^{11}$ to $4 \times 10^{12} \mathrm{~cm}^{-2}$, the barrier heights asymptotically approach the value of the corresponding de- fect energy (dashed lines in Fig. 3). For the interface defects close to the conduction band $\left(E_{\mathrm{it}}-E_{V}=880 \mathrm{meV}\right)$, we therefore find type inversion when $N_{\text {it }} \geq 8 \times 10^{11} \mathrm{~cm}^{-2}$. The band diagram shown in Fig. 2(a) corresponds to such a situation under equilibrium conditions. Figure 2(b) shows the same configuration under illumination and demonstrates that the capture of minority carriers into the interface states significantly reduces $Q_{\mathrm{it}}$ and, consequently, the band bending around the GB.

The reduction of band bending and that of the GB barrier $\Phi_{b}$ by illumination is demonstrated more quantitatively by the open symbols in Fig. 3 especially for the trap energies $E_{\mathrm{it}}-E_{V}=880 / 605 \mathrm{meV}$ whereas the curve with $E_{\mathrm{it}}-E_{V}$ $=270 \mathrm{meV}$ is barely affected by illumination. This latter situation appears to be the most probable in current CIGS cells, since, assuming a Fermi energy $160 \mathrm{meV}$ above valence band edge, $E_{\mathrm{it}}-E_{V}=270 \mathrm{meV}$ would yield a barrier of $q V_{b}=110 \mathrm{meV}$, in accordance with the available experimental data. ${ }^{6-9,12}$

\section{B. Recombination via grain boundaries}

Next, we turn to the consequences of GBs on the calculated photovoltaic output parameters. Figure 4 shows the efficiency $\eta$, short circuit current density $J_{\mathrm{SC}}$, and open circuit voltage $V_{\text {OC }}$ as a function of the GB defect density $N_{\text {it }}$ in a CIGS solar cell with grain size $g=2 \mu \mathrm{m}$. The base of these calculations is CIGS with a concentration $N_{\mathrm{bt}}$ of bulk defects $N_{\mathrm{bt}}=2 \times 10^{15} \mathrm{~cm}^{-3}$ and capture cross sections $\sigma_{\mathrm{bt}, n / p}$ $=10^{-15} \mathrm{~cm}^{2}$ corresponding to a bulk minority carrier lifetime of $5 \times 10^{-8} \mathrm{~s}$ and a diffusion length $L_{D}=3.6 \mu \mathrm{m}$. Consequently, the photovoltaic performance of the reference computation for an interface trap density $N_{\mathrm{it}}=0$ yields a high efficiency $\eta \approx 20 \%$. The influence of GBs is now modeled by increasing the density of GB defects with capture cross sections $\sigma_{\mathrm{it}, n / p}=10^{-15} \mathrm{~cm}^{2}$ at three different energies $E_{\mathrm{it}}$ $=E_{V}+270,605$, and $880 \mathrm{meV}$. The device efficiency decreases by increasing the number of GB defects, independently of the energy position of the traps, obeying an augmented recombination of carriers. Although this is the expected overall consequence of increasing $N_{\text {it }}$, an exceptional trend takes place for the photocurrent density [Fig. $4(\mathrm{~b})]$ in cells with traps at the energy $E_{\mathrm{it}}=E_{V}+880 \mathrm{meV}$, where $J_{\mathrm{SC}}$ is recovered beyond a minimum at $N_{\mathrm{it}} \approx 8$ $\times 10^{11} \mathrm{~cm}^{-2}$. Our calculations reveal that the reincrease in $J_{\text {SC }}$ toward the maximum available value at higher GB defect densities corresponds to the onset of type inversion at the GB under illumination. In this situation, the number of available holes limits GB recombination, and an increase in band bending at the GB reduces the recombination probability of electrons and, as a consequence, increases their collection probability.

However, the open circuit voltages $V_{\mathrm{OC}}$ displayed in Fig. 4(c) unveil that this specific improvement is restricted to the short circuit situation whereas under open circuit, recombination monotonically increases with increasing defect densities for all defect energies. This fact also dominates the overall performance shown in Fig. 4(a), demonstrating that the introduction of GB defects into a high quality CIGS absorber 
material leads to a uniform decline of the efficiency $\eta$ regardless of the assumed GB defect energy $E_{\mathrm{it}}$.

\section{Influence of grain size}

Efficiencies in excess of $19 \%$ for CIGS solar cells are possible with grain sizes of only $g \approx 0.5 \mu \mathrm{m}$, as shown in Ref. 2. In order to investigate the possibilities for such a high performance at such low grain sizes, we simulate cells with a vertical GB and a grain width $g=0.5 \mu \mathrm{m}$, for different trap energies and increasing defect densities. Figure 5 shows the efficiency depending on $N_{\text {it }}$ for $g=0.5 \mu \mathrm{m}$ (open symbols), and, for comparison, we include the results for $g=2 \mu \mathrm{m}$ (full symbols) extracted from Fig. 4(a). The bulk and GB defect energy is varied from $E_{\mathrm{it}}-E_{V}=E_{\mathrm{bt}}-E_{V}=270 \mathrm{meV}$ [Fig. 5(a)], to $605 \mathrm{meV}$ [Fig. 5(b)], and $880 \mathrm{meV}$ [Fig. 5(c)].

As expected, the smaller grain size yields lower efficiencies, regardless of the trap position. More importantly, our model indicates that efficiencies of $\eta=19 \%$ require a very low defect density $N_{\mathrm{it}}=10^{11} \mathrm{~cm}^{-2}$ for $g=0.5 \mu \mathrm{m}$, whereas $N_{\mathrm{it}}=2 \times 10^{11} \mathrm{~cm}^{-2}$ is needed for the larger grains $(g$ $=2 \mu \mathrm{m})$. In these cases, the corresponding band bendings are around $q V_{b}=25 \mathrm{meV}$ (cf. Fig. 3). We note here that $\eta$ $\geq 19 \%$ are also possible when using $N_{\mathrm{it}}=4 \times 10^{12} \mathrm{~cm}^{-2}$ combined with electron capture cross sections $\sigma_{\mathrm{it}, n}$ $=10^{-18} \mathrm{~cm}^{-2}$, i.e., much smaller than $\sigma_{\mathrm{it}, n / p}=10^{-15} \mathrm{~cm}^{-2}$ used in Fig. 5. In this case (not shown), the band bending amounts to $q V_{b}=100 \mathrm{meV}$.

\section{Spatial distribution of defects}

Up until this point, our calculations started with a rather perfect bulk material such that the efficiency level of the solar cells without GBs was higher than the record efficiencies. ${ }^{1}$ Section III D investigates the interplay between bulk and GB defects. Figure 6 compares the influence of GB defects on the efficiency of solar cells having a high bulk minority carrier lifetime $\tau_{b}=\left(v_{\mathrm{th}} N_{\mathrm{bt}} \sigma_{\mathrm{bt}}\right)^{-1}=5 \times 10^{-8} \mathrm{~s}$ to the efficiency of cells with low $\tau_{b}=5 \times 10^{-10} \mathrm{~s}$. We show here the example with a defect energy $E_{\mathrm{it}}-E_{V}=605 \mathrm{meV}$ for the GB and $E_{\mathrm{bt}}-E_{V}=350 \mathrm{meV}$ for the bulk defects. Variations of $\tau_{b}$ are achieved by the choice of $N_{\mathrm{bt}}=2 \times 10^{15} \mathrm{~cm}^{-3}$ (open symbols) and $N_{\mathrm{bt}}=2 \times 10^{13} \mathrm{~cm}^{-3}$ (full symbols) with a constant capture cross section $\sigma_{\mathrm{bt}, n / p}=10^{-13} \mathrm{~cm}^{2}$. The different choices of $N_{\mathrm{bt}}$ defines rather different efficiency levels of $20 \%$ and $11.8 \%$ at low values of $N_{\mathrm{it}} \leq 2 \times 10^{9} \mathrm{~cm}^{-2}$. However, the increase in $N_{\text {it }}$ leads to a deterioration of the cell efficiencies that depends very much on the assumed capture cross sections $\sigma_{\mathrm{it}, n / p}=10^{-15} \mathrm{~cm}^{2}$ (squares) and $\sigma_{\mathrm{it}, n / p}$ $=10^{-13} \mathrm{~cm}^{2}$ (circles) at the GBs. At high defect densities $N_{\text {it }} \geq 10^{12} \mathrm{~cm}^{-2}$, the efficiencies are entirely determined by the GBs. Thus, Fig. 6 illustrates the transition between the extreme situations of a solar cell that is dominated by defects in the grain interior and a cell that is defined by its GB properties. In addition, Fig. 6 shows calculations for a deeper bulk defect $E_{\mathrm{bt}}-E_{V}=605 \mathrm{meV}$ (crosses) with $N_{\mathrm{bt}}=2$ $\times 10^{15} \mathrm{~cm}^{-3}$ and $\sigma_{\mathrm{bt}, n / p}=10^{-15} \mathrm{~cm}^{2}$ [i.e., data already shown in Fig. 5(b)]. We conclude from the comparison of these data with the results for $E_{\mathrm{bt}}-E_{V}=350 \mathrm{meV}$ (open squares, $N_{\mathrm{bt}}$
$=2 \times 10^{13} \mathrm{~cm}^{-3}$ and $\left.\sigma_{\mathrm{bt}}=10^{-13} \mathrm{~cm}^{2}\right)$ that the choice of the bulk defect energy is rather irrelevant as long as the lifetime $\tau_{b}$ is high.

In our next numerical experiment, we investigate the influence of the spatial distribution of defects on the photovoltaic output parameters, by simulating cells with defects rather homogeneously distributed in the bulk to cells where the defects are concentrated at the coordinate of the vertical GB. These simulations assume cells where the bulk and the GB defect level have equal capture cross sections and energy level, gradually increasing $N_{\mathrm{it}}$ and reducing $N_{\mathrm{bt}}$, thus maintaining the mean defect density $N_{m}$ according to

$$
N_{m}=N_{\mathrm{bt}}+N_{\mathrm{it}} / g \text {. }
$$

The case of $N_{\mathrm{it}}=0$ represents a monocrystalline material were all defects are homogeneously distributed in the bulk, whereas $N_{\mathrm{it}}=4 \times 10^{12} \mathrm{~cm}^{-2}$ corresponds to the polycrystalline material where all defects are located at the GB. Figure 7 shows the photovoltaic output parameters as a function of $N_{\text {it }}$ using $N_{m}=2 \times 10^{16} \mathrm{~cm}^{-3}$, for defect levels with energies $270 / 605 / 880 \mathrm{meV}$ (circles, squares, stars) above valence band. With capture cross sections $\sigma_{\mathrm{bt}, n / p}=\sigma_{\mathrm{it}, n / p}=10^{-14} \mathrm{~cm}^{2}$ (open symbols), the power conversion efficiency increases only in the case of $E_{\mathrm{it}}=270 \mathrm{meV}$, by $0.4 \%$ absolute when all defects are concentrated at the GB $\left(N_{\mathrm{it}}=4 \times 10^{12} \mathrm{~cm}^{-2}\right)$. Taking $\sigma_{\mathrm{bt}, n / p}=\sigma_{\mathrm{it}, n / p}=10^{-13} \mathrm{~cm}^{2}$ (closed symbols) results in an increase in the efficiency of around 3\% absolute for all three defect energies. This result with highly recombination active defects is consistent with the scaling law of Ref. 32 . This law states that at a given number of recombination centers the concentration of these defects in well separated cell areas (here, the GBs) is always a more favorable situation compared to a spatially homogeneous distribution of these defects. However, a benefit of defects concentrated at the GB instead of distributed in the bulk is guaranteed only in cases where the defects have sufficiently strong recombination activity. When defects are less active, as seen in Fig. 7(a) from the curves where $\sigma_{n / p}=10^{-14} \mathrm{~cm}^{-2}$, the shift of the defects from the bulk to the GB slightly rise the device efficiency only when the defect energy is lower than midgap (open circles), and harms the device performance if the defects lie near/above midgap (open squares and open stars).

\section{E. Collection of carriers along grain boundaries}

The enhancement of carrier collection along inverted grain boundaries has been discussed as one possible advantage of polycrystalline semiconductors with respect to their monocrystalline counterparts. From the experimental evidence it appears that type inversion applies rather to CdTe solar cells ${ }^{4,5}$ whereas in CIGS (Refs. 7-9, 12, 16, and 17) the band bending around the GB is relatively small. Hence, the following calculations aim more at a general understanding of carrier collection along GBs than to the real situation in CIGS.

As we have already seen in Fig. 4, an improvement of carrier collection by inverted GBs is overcompensated by the increase in GB recombination when taking a high performance CIGS bulk as a starting point. Therefore, we explore 


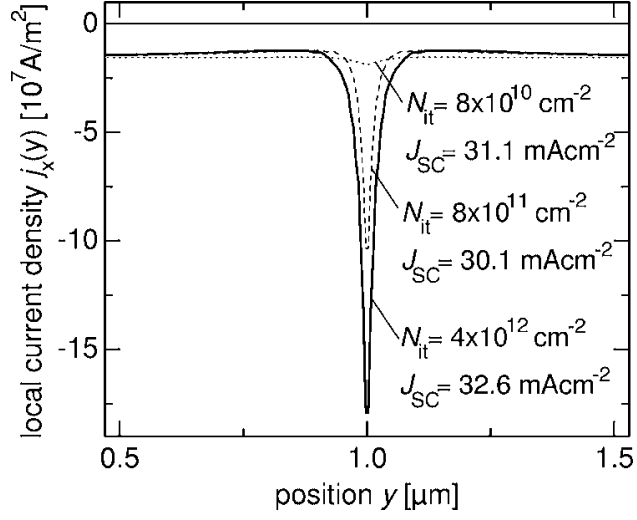

FIG. 9. Profiles of the vertical component of the local current density $j_{x}$ calculated at a depth of $100 \mathrm{~nm}$, i.e., close to the cell junction. The profiles are calculated under illumination, short circuit conditions, for grain boundary (GB) defect densities of $N_{\mathrm{it}}=8 \times 10^{10} / 8 \times 10^{11} / 4 \times 10^{12} \mathrm{~cm}^{-2}$.

now the situation of a rather mediocre bulk material. The assumed bulk defects have an energy $605 \mathrm{meV}$ above the valence band edge, a concentration $N_{\mathrm{bt}}=2 \times 10^{15} \mathrm{~cm}^{-3}$, and capture cross sections $\sigma_{\mathrm{bt}, n / p}=10^{-14} \mathrm{~cm}^{2}$, corresponding to a bulk diffusion length $L_{D} \approx 1.1 \mu \mathrm{m}$. The reference computation for a GB trap density $N_{\mathrm{it}}=0$ yields a photovoltaic performance of $\eta \approx 16 \%$ and $J_{\mathrm{SC}} \approx 31 \mathrm{~mA} \mathrm{~cm}^{-2}$, as shown in Fig. 8. Consistently, these values are somewhat lower than those obtained in Fig. 4 where smaller $\sigma_{\mathrm{bt}, n / p}=10^{-15} \mathrm{~cm}^{2}$ were taken. The introduction of GB defects at energy $E_{\mathrm{it}}$ $=E_{V}+880 \mathrm{meV}$ with capture cross sections $\sigma_{\mathrm{it}, n / p}$ $=10^{-14} / 10^{-15} / 10^{-16} \mathrm{~cm}^{2}$ leads to an enhancement of $J_{\mathrm{SC}}$ up to $32.6 \mathrm{~mA} \mathrm{~cm}{ }^{-2}$ at $N_{\mathrm{it}}=4 \times 10^{12} \mathrm{~cm}^{-2}$. However, an overall improvement of the efficiency $\eta$ is not observed, as the gains in $J_{\mathrm{SC}}$ are overcompensated by the losses in $V_{\mathrm{OC}}$. The trap density $N_{\text {it }}=4 \times 10^{12} \mathrm{~cm}^{-2}$ corresponds to an addition of 2 $\times 10^{16} \mathrm{~cm}^{-3}$ to the total defect concentration. In the case of low capture cross section $\sigma_{\mathrm{it}, n / p}=10^{-16} \mathrm{~cm}^{2}$ (circles in Fig. 8 ), this adds roughly only $10 \%$ to the recombination in the grain interior with $N_{\mathrm{bt}}=2 \times 10^{15} \mathrm{~cm}^{-3}$ and $\sigma_{\mathrm{bt}, n / p}$ $=10^{-14} \mathrm{~cm}^{2}$. Therefore, we conclude that losses in $V_{\text {OC }}$ are a combination of the additional GB recombination and a higher recombination through bulk defects in the spacecharge region surrounding the GB. Even considering cases where the carrier mobilities are an order of magnitude lower (results not shown here) and the different combinations of bulk and GB capture cross sections used above, we have not identified a situation where the introduction of an inverted GB in the way described here leads to an enhanced efficiency of the solar cell.

The improvement in $J_{\mathrm{SC}}$ seen in Fig. 8(b) does only weakly depend on the chosen capture cross section, indicating that carrier collection is effectively enhanced by the GB provided sufficient type inversion is achieved. Interestingly, for intermediate concentrations and the larger capture cross sections, the $J_{\mathrm{SC}}$ data exhibit a minimum, pointing to a nonnegligible effect of GB recombination on carrier collection. To shed some light into the enhancement of $J_{\mathrm{SC}}$ with increasing $N_{\text {it }}$, we calculate spatial profiles of the perpendicular current density component $j_{x}$ close to the heterojunction ( $x$ $=100 \mathrm{~nm}$ ). These profiles (shown in Fig. 9) correspond to the simulations of Fig. 8(b) belonging to the curve with $\sigma_{\mathrm{it}, n / p}=10^{-15} \mathrm{~cm}^{2}$ for $N_{\mathrm{it}}=8 \times 10^{10}$ (dotted) $/ 8$ $\times 10^{11}$ (dashed) $/ 4 \times 10^{12}$ (continuous) $\mathrm{cm}^{-2}$. For clarity, the curves are displayed in the interval $0.5 \mu \mathrm{m}<y$ $<1.5 \mu \mathrm{m}$. At $N_{\mathrm{it}}=8 \times 10^{11} \mathrm{~cm}^{-2}$ (dashed curve), the cell operates at the onset of type inversion at the GB. All three profiles reflect that with increasing band bending (higher $N_{\text {it }}$ ) the current density becomes increasingly concentrated at the GB. However, this current crowding effect leads to higher $J_{\mathrm{SC}}$ values only if the recombination probability around the GB is lower than in the bulk. Such a condition is only achieved when sufficient-type inversion in the surroundings of the GB is attained. This explains that the dashed curve in Fig. 9 yields the lowest $J_{\mathrm{SC}}$ value, since at the onset of inversion, the carrier concentrations at the GB are nearly equal, yielding maximum recombination rates. The case with $N_{\text {it }}$ $=4 \times 10^{12} \mathrm{~cm}^{-2}$ (continuous curve in Fig. 9) has a much stronger inverted population around the GB, which result in an improved $J_{\mathrm{SC}}$.

\section{EFFECT OF THE INTERNAL BAND OFFSET}

\section{A. Excess barrier height}

It has been suggested in the past that one important ingredient for the high performance of polycrystalline CIGS thin-film solar cells stems from an internal valence band offset $\Delta E_{V}$ at the GBs (sometimes also referred to as a neutral barrier) resulting from a slightly $\mathrm{Cu}$-poor composition of the region adjacent to the GB. ${ }^{13-15}$ The effect of this internal offset at the GBs could be of similar importance as it is at the surface of the absorber, ${ }^{33}$ whereas, there is ample experimental evidence for the $\mathrm{Cu}$-poor surface layer ${ }^{34,35}$ and its beneficial consequences for the performance of CIGS solar cells as long as the overall film composition is $\mathrm{Cu}$-poor, ${ }^{36}$ the question whether or not such a $\mathrm{Cu}$-poor layer is a general positive feature of GBs in CIGS is still under discussion. ${ }^{37-40}$

The present study concentrates on investigating the effect of such an internal band offset and on finding the conditions that have to be fulfilled if the $\mathrm{Cu}$-poor layer should have a decisive beneficial effect on the performance of polycrystalline CIGS solar cells. Figure 10 shows the band diagram of a $\mathrm{GB}$ surrounded by a $\mathrm{Cu}$-poor layer that has a valence band offset $\Delta E_{V}$ toward the CIGS bulk. In such a situation, the hole barrier at the GB is enhanced leading to an effective barrier $\Phi_{b}^{*}=\Phi_{b}+\Delta E_{V}$. Note that only the part $\Phi_{b}$ results from the interface charges $Q_{\text {it }}$ whereas the internal valence band offset $\Delta E_{V}$ leads to an additional contribution that is sometimes referred to as neutral barrier. ${ }^{14,17}$

This additional barrier prevents holes from the grain interior to reach the location of the GB. As long as the GB is parallel to the direction of carrier transport, we can assume that the band offset does not affect carrier transport in the direction to the collecting junction. Then, the effect of the internal band offset is the reduction of carrier recombination. In the following, we model recombination at the GB with the help of a reduced effective hole capture cross section $\sigma_{\mathrm{it}, p}^{\text {eff }}$, which in the Appendix is shown to obey 

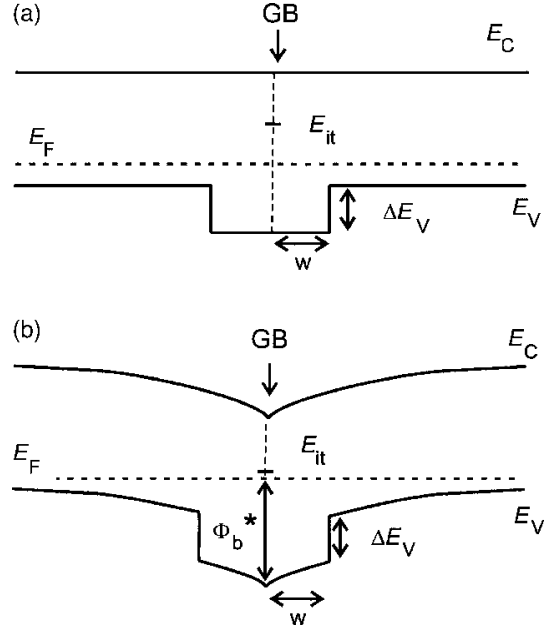

FIG. 10. Band diagram across an uncharged, flatband GB displaying an internal valence band offset of $\Delta E_{V}$ at the location of the grain boundary (a), and across a charged GB (b) with a band bending $q V_{b}$. The energy of the defect level at the GB is $E_{\mathrm{it}}$

$$
\sigma_{\mathrm{it}, p}^{\mathrm{eff}}=\sigma_{\mathrm{it}, p} \exp \left(-\Delta E_{V} / k T\right),
$$

where $k T$ denotes the thermal energy. Note that according to Eq. (7), one order of magnitude in the ratio $r_{\sigma}=\sigma_{\mathrm{it}, p}^{\text {eff }} / \sigma_{\mathrm{it}, p}$ corresponds almost exactly to an increment of the band offset $\Delta E_{V}$ by $60 \mathrm{meV}$ at room temperature.

Let us first assume a high GB defect concentration $\left(N_{\mathrm{it}}\right.$ $=4 \times 10^{12} \mathrm{~cm}^{-2}$ ), serving as limiting cases for maximum GB recombination activity, to gain a general overview on the effect of the internal band offset. Figure 11 compares the conversion efficiency $\eta$, short circuit current densities $J_{\mathrm{SC}}$, and open circuit voltages $V_{\mathrm{OC}}$ as a function of the ratio $r_{\sigma}$, assuming $\sigma_{\mathrm{it}, p}=\sigma_{\mathrm{it}, n}=10^{-15} \mathrm{~cm}^{2}$. These simulations use bulk defect densities $N_{\mathrm{bt}}=2 \times 10^{15} \mathrm{~cm}^{-3}$, and bulk capture cross sections $\sigma_{\mathrm{bt}, n / p}=10^{-15} \mathrm{~cm}^{2}$. The defect energies are assumed equal in the bulk and at the $\mathrm{GB}$, being $E_{\mathrm{it}}=E_{\mathrm{bt}}=E_{t}$

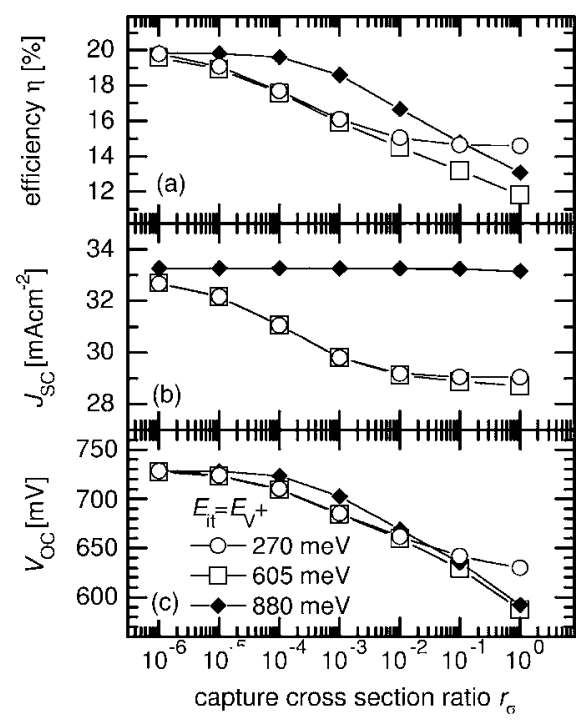

FIG. 11. Simulated efficiency $\eta$, short circuit current density $J_{\mathrm{SC}}$, and open circuit voltage $V_{\mathrm{OC}}$ as a function of the ratio $r_{\sigma}$ between effective hole and electron capture cross sections at the GB. Note that one order of magnitude in $r_{\sigma}$ corresponds to an increment of the band offset $\Delta E_{V}$ by $60 \mathrm{meV}$. The defect density at the grain boundary is $4 \times 10^{12} \mathrm{~cm}^{-2}$.

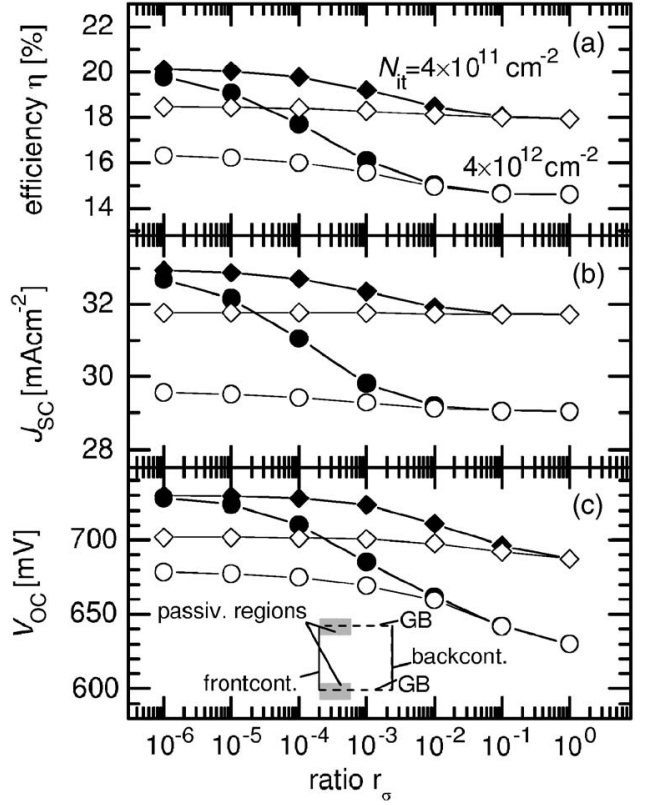

FIG. 12. Simulated efficiency $\eta$, short circuit current density $J_{\mathrm{SC}}$, and open circuit voltage $V_{\mathrm{OC}}$ as a function of the ratio $r_{\sigma}$ between effective hole and electron capture cross sections at the GB, for increasing grain boundary defect densities $N_{\text {it }}$. The defect energy is $270 \mathrm{meV}$ above the valence band edge for bulk as well as for grain boundary defects. Open symbols stand for the case where the internal valence band offset extends along the hole length of the GB. Full symbols correspond to the situation where only the first third $(0.66 \mu \mathrm{m})$ of the GB is passivated by the band offset as shown in the inset.

$=270 \mathrm{meV}$ (circles) $/ 605 \mathrm{meV}$ (squares) $/ 880 \mathrm{meV}$ (diamonds). All energy values are taken relative to the valence band maximum in the grain.

As shown in Fig. 11, decreasing values of the cross section ratio $r_{\sigma}$ improve $\eta$, independently of $E_{\mathrm{it}}$. Comparing the efficiency values at the extreme values of $r_{\sigma}, \eta$ increases between $5 \%$ and $8 \%$ absolute (depending on the defect energy) when $r_{\sigma}$ is reduced from $10^{\circ}$ (equal capture cross sections, no GB band offset) to $10^{-6}$, corresponding to a band offset of $\Delta E_{V}=360 \mathrm{meV}$. Although $V_{\text {OC }}$ increases with decreasing $r_{\sigma}$ independently of $E_{\mathrm{it}}$, the short circuit current density shown in Fig. 11(b) drastically changes its behavior when $E_{\text {it }}$ lies above midgap. Indeed, the simulations with $E_{\mathrm{it}}=E_{V}+880 \mathrm{meV}$ show a high $J_{\mathrm{SC}}=33 \mathrm{~mA} \mathrm{~cm}^{-2}$ already at $r_{\sigma}=10^{\circ}$, with no influence of $r_{\sigma}$. This feature explains that the efficiency shown in Fig. 11(a) tends to be higher for the defect above midgap than for defects near or below midgap (squares, circles). This effect is explained by the phenomenon of improved carrier collection by inverted GB, guaranteed by the high defect density $N_{\mathrm{it}}=4 \times 10^{12} \mathrm{~cm}^{-2}$.

In the next step, we deepen our analysis for the case of a rather shallow defect level in combination with band offsets, and intermediate defect densities. Figure 12 shows the output parameters as a function of $r_{\sigma}$, obtained for $E_{\mathrm{it}}=E_{V}$ $+270 \mathrm{meV}$ and $N_{\mathrm{it}}=4 \times 10^{11}$ and $4 \times 10^{12} \mathrm{~cm}^{-2}$. The data points with $N_{\mathrm{it}}=4 \times 10^{12} \mathrm{~cm}^{-2}$ (open circles) are the same as in Fig. 11 (open circles). We see that at the lowest ratio $r_{\sigma}$ $=10^{-6}$, corresponding to $\Delta E_{V}=360 \mathrm{meV}$, all effects of GB recombination are eclipsed. Figure 12 shows that high efficiencies around $20 \%$ are possible for $N_{\text {it }}<2 \times 10^{12} \mathrm{~cm}^{-2}$, and ratios $r_{\sigma} \leq 10^{-5}$, i.e., band offsets $\Delta E_{V} \geq 300 \mathrm{meV}$. 
Additionally, Fig. 12 shows the calculated parameters (full symbols) under the assumption that the GB is passivated only to one-third of its length toward the front surface by the internal band offset as sketched by the inset in Fig. 12 (c). Such an assumption is reasonable for two reasons. First, there is ample evidence that the $\mathrm{Cu}$-poor region providing the internal valence band offset is present at the surface of the absorber. ${ }^{34-36}$ It is therefore reasonable to conclude that a $\mathrm{Cu}$-poor region at the GBs is more likely to be present toward the surface of the absorber than toward the back contact. Second, measurements of the majority-carrier transport properties of CIGS films in coplanar geometry, i.e., perpendicular to many GBs, exhibit activation energies in a range between 60 and $120 \mathrm{meV}^{41}$ i.e., much smaller than an effective internal band offset. Therefore, the extra barrier cannot be present along the full length of all grains in the CIGS films. Rather, it might be present at some GBs and/or only for a part of the GB length.

A good passivation of GBs for the first $0.66 \mu \mathrm{m}$ should be a relatively effective situation because at least recombination in the space charge region is reduced. This is reflected in the fact that $V_{\mathrm{OC}}$ moderately increases with decreasing $r_{\sigma}$ (increasing $\Delta E_{V}$ ) for the partially passivated GBs in Fig. $12(\mathrm{c})$. However, $J_{\mathrm{SC}}$ remains essentially unaffected by the increase in the internal barrier [Fig. 12(b)] resulting eventually in rather moderate improvements of the efficiency [Fig. 12(a)]. We conclude from this result that not only a high barrier height of at least $300 \mathrm{meV}$ but also an almost homogeneous presence of the $\mathrm{Cu}$-poor layer around the GBs along their entire length is required to virtually eclipse their deleterious impact on the device performance.

\section{B. Influence of the barrier width}

It is evident that a mere interface reconstruction is not sufficient to provide a sufficient interface passivation because holes may penetrate the extra barrier by quantummechanical tunneling. Therefore, only a sufficiently thick high band gap layers will prevent holes from tunneling into the interface states at the GBs. In the following, we investigate the tunneling effect considering a rectangular energy barrier with an energy height $\Delta E_{V}$ around the GB. The probability $P_{\text {Tun }}$ to penetrate such a barrier is found in the WKB expression $^{42}$

$$
P_{\text {Tun }}=\exp \left(\frac{-2 w\left(2 m^{*} \Delta E_{V}\right)^{1 / 2}}{\hbar}\right),
$$

where $w$ is the width of the barrier, i.e., the width of the $\mathrm{Cu}$-poor wide-gap region on either side of the GB (cf. Fig. 10). The quantity $\hbar$ is Planck's constant and $m^{*}$ denotes the effective mass of the hole in the $\mathrm{Cu}$-poor interface layer (assumed to be 0.7 times the free electron mass $m_{e}$ according to Ref. 43). Figure 13 displays the tunneling probability $P_{\text {Tun }}$ (dotted lines) and the sum $P=P_{\mathrm{Tun}}+P_{\mathrm{TE}}$ (solid lines) that corresponds to the probability that a hole from the bulk of the grain arrives at the GB either by tunneling or by thermionic emission with the probability $P_{\mathrm{TE}}=\exp \left(-\Delta E_{V} / k T\right)$ (dashed lines in Fig. 13). As can be seen from Fig. 13, a barrier width $w>3 \mathrm{~nm}$ is needed to reduce the tunneling

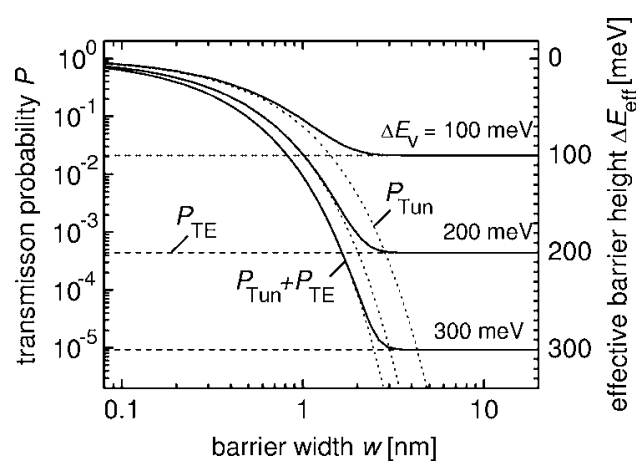

FIG. 13. Tunneling probability $P_{\text {Tun }}$ (dotted lines), thermionic-emission probability $P_{\mathrm{TE}}$ (dashed lines) and combined probability $P=P_{\mathrm{Tun}}+P_{\mathrm{TE}}$ for carriers trespassing an energy barrier of width $w$ and height $\Delta E_{V}$ $=100 / 200 / 300 \mathrm{meV}$. Roughly, beyond a barrier width $w>3 \mathrm{~nm}$, tunneling becomes negligible, remaining thermionic emission as the only transmission mechanism. The right axis indicates the effective barrier height $\Delta E_{\text {eff }}$ (see Sec. VG for details).

probability to negligible values and, in consequence, the probability $P$ to the thermionic-emission values given by Eq. (7). Note here that the transmission probability enters into our numerical simulations via the capture cross section ratio $r_{\sigma}=P$. Therefore, all results in Figs. 11 and 12 account either for a specific valence band offset together with a sufficient thickness $w \geq 3 \mathrm{~nm}$ or for a given combination of $w$ $\leq 3 \mathrm{~nm}$ and $\Delta E_{V}$ where the resulting $r_{\sigma}$ must be extracted from Fig. 12. Thus, we conclude that an almost perfect passivation of the GBs resulting from $r_{\sigma} \leq 10^{-5}$ in Figs. 10 and 11 not only requires a considerable band offset $\Delta E_{V}$ $\geq 300 \mathrm{meV}$ but also a significant width $w \geq 3 \mathrm{~nm}$ of the $\mathrm{Cu}$-poor region on either side of the GB.

At this point, it is important to note that the considerations above are also valid for the passivation effect of the $\mathrm{Cu}$-poor layer at the surface of CIGS films. This layer has a thickness that varies between 5 and $60 \mathrm{~nm}$ depending on the overall film composition. ${ }^{44}$ Hence, tunneling could reduce the passivation effect of this surface layer in case of CIGS films that come very close to a stoichiometrical composition. Ensuring a sufficient thickness of the $\mathrm{Cu}$-poor surface layer seems therefore of importance for high-efficiency CIGS solar cells. We argue that the last ( $\mathrm{Cu}$-free) deposition stage of the three-stage deposition process ${ }^{45}$ for highest efficiency CIGS solar cells plays an important role in this context.

\section{HORIZONTAL GRAIN BOUNDARIES}

Grains in high-efficiency CIGS absorbers are preferentially columnar making the vertical GB model the most important one. However, GBs parallel to the cell's surface are present to a certain amount in all CIGS solar cells. This section investigates the impact of such horizontal GBs on the cell performance, according to the geometry sketched in Fig. 1 , where the GB is placed at a depth $x=1 \mu \mathrm{m}$. Here, we are particularly interested in the effect of the hole barrier, since it introduces an additional physical effect compared to the situation of the vertical GB: the action as an obstacle to carrier transport.

The effect of potential barriers on majority-carrier transport is usually modeled by lowering the carrier concentration 


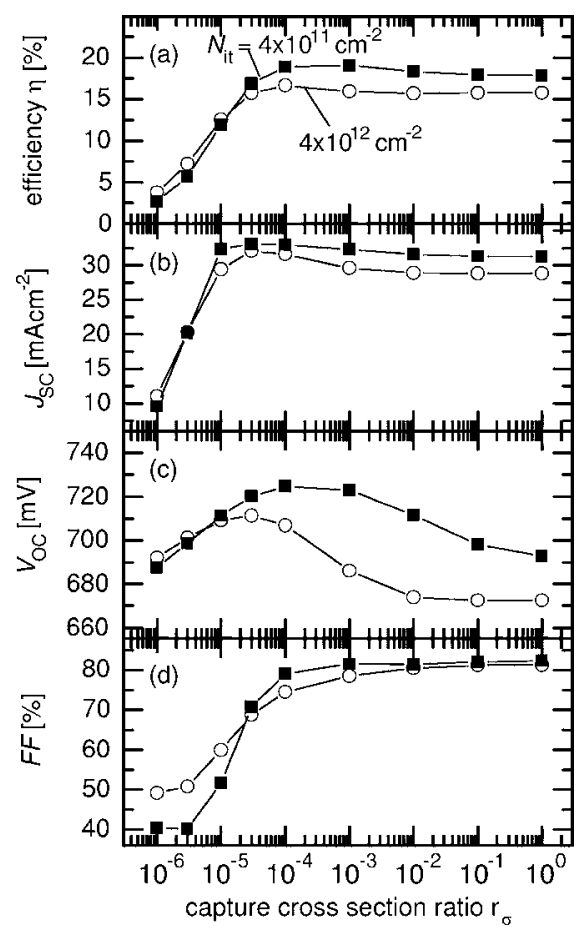

FIG. 14. Simulated solar cell parameters as a function of the ratio $r_{\sigma}$ between effective hole and electron capture cross sections at the GB, for a cell with a horizontal GB located at the middle of the absorber. Note that no vertical GBs are present in these calculations. The curves are calculated for GB defect densities $N_{\text {it }}=4 \times 10^{11} / 4 \times 10^{12} \mathrm{~cm}^{-2}$ (squares/circles). The defect energy is $270 \mathrm{meV}$ above the valence band edge for bulk as well as for GB defects. The introduction of a hole barrier to the horizontal GB is beneficial only up to about $r_{\sigma}=10^{-3}-10^{-4}$. Below this range, the fill factor (FF) falls rapidly, followed by additional drops in $J_{\mathrm{SC}}$ and $V_{\mathrm{OC}}$, with the expected drop of cell efficiency $\eta$.

within the barrier by the factor $\exp \left(-\Delta E_{V} / k T\right)$, or by keeping the carrier concentration constant and reducing the mobility by the same factor. ${ }^{46}$ Here, we follow the latter strategy by assuming an effective hole mobility given by

$$
\mu_{p}^{\text {eff }}=\mu_{p} \exp \left(-\Delta E_{V} / k T\right)=\mu_{p} r_{\sigma}
$$

in the surroundings $( \pm 20 \mathrm{~nm})$ of the GB.

The calculated solar cell output parameters as a function of $r_{\sigma}$ are shown in Fig. 14. The curves are calculated for two GB defect densities $N_{\text {it }}$ that differ by one order of magnitude, $4 \times 10^{11} \mathrm{~cm}^{-2}$ (squares), and $4 \times 10^{12} \mathrm{~cm}^{-2}$ (circles). For bulk and GB defects, a defect energy of $270 \mathrm{meV}$ above the valence band edge and equal electron and hole capture cross sections $\sigma_{\mathrm{bt}, n / p}=\sigma_{\mathrm{it}, n / p}=10^{-15} \mathrm{~cm}^{2}$ are assumed. Note that $n o$ vertical $\mathrm{GB}$ is assumed in the following calculations. As seen in the efficiency $\eta$ [Fig. 14(a)], the introduction of a hole barrier to the horizontal GB is beneficial only up to $r_{\sigma}$ $=10^{-3}-10^{-4}$, depending on $N_{\text {it }}\left(\Delta E_{V}=180-240 \mathrm{meV}\right)$. In this regime, the hole barrier acts similarly to the case of the vertical GB described previously, i.e., by reducing the recombination probability for holes. The overall efficiency improvement in this regime is due to an enhancement of the open circuit voltage [Fig. 14(c)]. At $r_{\sigma}$ below $10^{-4}$, the barrier hinders carrier transport and, therefore, strongly reduces the short circuit current density $J_{\mathrm{SC}}$, the fill factor (FF), and consequently, the cell efficiency $\eta$. Especially, the fill factor FF shown in Fig. 14(d) monotonically decreases with de- creasing $r_{\sigma}$. By comparing the values at the extremes of the $r_{\sigma}$ axis, we notice that at $r_{\sigma}=10^{-6}, J_{\mathrm{SC}}$ and FF drop to about $1 / 3$ and $1 / 2$ of their values at $r_{\sigma}=10^{\circ}$, respectively.

The results shown in Fig. 14 demonstrate that cell regions with horizontal GBs with a hole barrier that is wide and high enough to prevent recombination implies that hole transport across the same barrier becomes almost impossible. Especially for values $\Delta E_{V}>240 \mathrm{meV}$ where the reduction of carrier recombination becomes significant (cf. Figs. 11 and 12), an additional horizontal GB virtually eclipses the (local) short circuit current that would originate from light absorption in the front part of the grain.

\section{SUMMARY}

The present paper investigates GB effects on the performance of CIGS solar cells, studying the effects of defect densities, energy levels and capture cross sections, grain size, and GB orientation (vertical and horizontal to the cell's surface). Our results show that the introduction of GB defects results in an overall efficiency loss from $20 \%$ to below $12 \%$, making proper passivation of GBs a primary requirement for high efficiency. In order to reach $\eta=19 \%$ with electron and hole capture cross sections of $10^{-15} \mathrm{~cm}^{-2}$ at vertical GBs, our results predict that low defect densities of $N_{\text {it }}$ $=10^{11} \mathrm{~cm}^{-2}$ for a grain size of $g=0.5 \mu \mathrm{m}$, and $N_{\mathrm{it}}=2$ $\times 10^{11} \mathrm{~cm}^{-2}$ for $g=2 \mu \mathrm{m}$ are required. Such low defect densities yield negligible GB barrier heights. In order to reproduce the experimentally found GB barrier heights of 100 $\mathrm{meV}$ reported in the literature, our calculations require $\mathrm{GB}$ defect densities of $2 \times 10^{12} \mathrm{~cm}^{-2}$, considering a defect level at $270 \mathrm{meV}$ above valence band edge. In such a situation, efficiencies of $\eta=19 \%$ would require an electron capture cross section of $10^{-18} \mathrm{~cm}^{2}$ (at the vertical GB).

Highly concentrated defects placed in the upper half of the band gap yield inverted GBs. Such inverted GBs improve the collection of photogenerated carriers in the cell, enhancing the short current density $J_{\mathrm{SC}}$. However, $\eta$ is not improved because this effect is counterbalanced by losses in $V_{\mathrm{OC}}$ associated with the required high defect concentration. Only when comparing monocrystalline and polycrystalline CIGS with the same overall number of defects we observe that redistributing these defects toward the GBs may improve the device performance especially for high defect concentrations and/or high defect capture cross sections. However, efficiencies $\eta$ in excess of $16 \%$ are hardly compatible with a significant concentration of defects close to the conduction band and with inverted GBs.

We further simulate cells featuring a band offset in the valence band at the GB, a hole barrier, as a possible efficient passivation mechanism working at the GBs. ${ }^{13,14}$ Barriers at least $300 \mathrm{meV}$ high would effectively reduce recombination at the GB provided the barrier is present along the entire GB. Additionally, a minimum thickness of $3 \mathrm{~nm}$ is needed to prevent quantum-mechanical tunneling of carriers into the GB. However, barriers that fulfill these requirements for width and height are no longer beneficial when associated with horizontal GBs, where they act as obstacle for hole transport across the GBs. Thus, the hole barrier may play an 
important role in CIGS films by passivating some, possibly the most recombination active, GBs. However, this barrier cannot be a general feature of all GBs in high-efficiency CIGS absorbers. The generally benign character of GBs must also embrace beneficial crystallographic GB structures $^{11,12}$ and defect chemical passivation of GB defects ${ }^{18-21}$ in order to keep the defect density low.

\section{ACKNOWLEDGMENTS}

The authors are grateful to T. Kirchartz and J. H. Werner for collaboration and discussions. We also acknowledge $\mathrm{M}$. Troviano (UNC) and B. Pieters (FZJ) for a critical reading of this manuscript.

\section{APPENDIX: DERIVATION OF EFFECTIVE CAPTURE CROSS SECTIONS}

Here, we obtain Eq. (7) considering the band diagram shown in Fig. 10(a), although the resulting expression is perfectly valid also for a charged grain boundary as in Fig. 10(b). By using the expression for the SRH recombination rate evaluated at the location of the $\mathrm{GB}$, we demonstrate that the incorporation of a valence band offset $\Delta E_{V}$ at the GB alters the equation only by the hole capture cross section, which must be replaced by the effective capture cross section $\sigma_{\text {it, } p}^{\text {eff }}$ given by Eq. (7). The recombination rate given by Eq. (3), depends on the electron and hole concentrations at the $\mathrm{GB}$, given by $n_{\mathrm{it}}=N_{C} \exp \left[\left(E_{F n}-E_{C}\right) / k T\right], p_{\mathrm{it}}=N_{V} \exp \left[\left(E_{V}\right.\right.$ $\left.\left.-E_{F p}\right) / k T\right]$, where $E_{F n}$ and $E_{F p}$ are the electron and hole quasi-Fermi energy levels, respectively. When a valence band offset $\Delta E_{V}$ is added, Eq. (3) must be rewritten using the following replacements:

$$
\begin{aligned}
& p_{\text {it }} \rightarrow p_{\text {it }} \exp \left(-\Delta E_{V} / k T\right), \\
& p_{1, \text { it }} \rightarrow p_{1, \text { it }} \exp \left(-\Delta E_{V} / k T\right) .
\end{aligned}
$$

By performing these replacements, and considering that under solar cell operation $n_{i}^{2}$ is negligible, the recombination rate is given by

$$
R_{\mathrm{it}}=\frac{N_{\mathrm{it}} v_{\mathrm{th}} n_{\mathrm{it}} p_{\mathrm{it}}}{\sigma_{\mathrm{it}, p}^{-1} \exp \left(\Delta E_{V} / k T\right)\left(n_{\mathrm{it}}+n_{1, \mathrm{it}}\right)+\sigma_{\mathrm{it}, n}^{-1}\left(p_{\mathrm{it}}+p_{1, \mathrm{it}}\right)} .
$$

This expression indicates that when adding a valence band offset, $R_{\mathrm{it}}$ may be calculated as if there where no offset, but considering a hole effective capture cross section given by

$$
\sigma_{\mathrm{it}, p}^{\mathrm{eff}}=\sigma_{\mathrm{it}, p} \exp \left(-\Delta E_{V} / k T\right) .
$$

Since the recombination rate analyzed here is at the location of the GB, this expression is also valid for charged GBs with a band bending, as shown in Fig. 10(b).

${ }^{1}$ K. Ramanathan, M. A. Contreras, C. L. Perkins, S. Asher, F. S. Hasoon, J. Keane, D. Young, M. Romero, W. Metzger, R. Noufi, J. Ward, and A. Duda, Prog. Photovoltaics 11, 225 (2003).

${ }^{2}$ P. Jackson, R. Würz, U. Rau, J. Mattheis, M. Kurth, T. Schlötzer, G. Bilger, and J.-H. Werner, Prog. Photovoltaics 15, 507 (2007).

${ }^{3}$ Active area efficiency of a CuInSe ${ }_{2}$ cell reported by C. H. Champness, $\mathrm{H}$. $\mathrm{Du}$, and I. Shih, Proceedings of the 29th IEEE Conference (IEEE, Piscataway, New York, 2002), p. 732. We notice that this cell uses a simple, unoptimized front contact (strongly suffering from series resistance), and no antireflection coating.
${ }^{4}$ I. Visoly-Fisher, S. R. Cohen, A. Ruzin, and D. Cahen, Adv. Mater. (Weinheim, Ger.) 16, 879 (2004).

${ }^{5}$ I. Visoly-Fisher, S. R. Cohen, A. Ruzin, and D. Cahen, Adv. Funct. Mater. 16, 649 (2006).

${ }^{6}$ S. Schuler, S. Nishiwaki, J. Beckmann, N. Rega, S. Brehme, S. Siebentritt, and M. Ch. Lux-Steiner, Proceedings of the 29th IEEE Photovoltaic Specialists Conference (IEEE, Piscataway, 2002), p. 504.

${ }^{7}$ S. Siebentritt and S. Schuler, J. Phys. Chem. Solids 64, 1621 (2003).

${ }^{8}$ S. Sadewasser, Th. Glatzel, S. Schuler, S. Nishiwaki, R. Kaigawa, and M. Ch. Lux-Steiner, Thin Solid Films 431-432, 257 (2003).

${ }^{9}$ T. Meyer, F. Engelhardt, J. Parisi, and U. Rau, J. Appl. Phys. 91, 5093 (2002).

${ }^{10}$ M. J. Romero, K. Ramanathan, M. A. Contreras, M. M. Al-Jassim, R. Noufi, and P. Sheldon, Appl. Phys. Lett. 83, 4770 (2003).

${ }^{11}$ N. Ott, G. Hanna, U. Rau, J. H. Werner, and H. P. Strunk, J. Phys.: Condens. Matter 16, S85 (2004).

${ }^{12}$ G. Hanna, T. Glatzel, S. Sadewasser, N. Ott, H. P. Strunk, U. Rau, and J. H. Werner, Appl. Phys. A: Mater. Sci. Process. 81, 1 (2006).

${ }^{13}$ C. Persson and A. Zunger, Phys. Rev. Lett. 91, 266401 (2003).

${ }^{14}$ C. Persson and A. Zunger, Appl. Phys. Lett. 87, 211904 (2005).

${ }^{15}$ M. J. Hetzer, Y. M. Strzhemechny, M. Gao, M. A. Contreras, A. Zunger, and L. J. Brillson, Appl. Phys. Lett. 86, 162105 (2005).

${ }^{16}$ D. Azulay, O. Millo, I. Balberg, H.-W. Schock, I. Visoly-Fisher, and D. Cahen, Sol. Energy Mater. Sol. Cells 91, 85 (2007).

${ }^{17}$ S. Siebentritt, S. Sadewasser, M. Wimmer, C. Leendertz, T. Eisenbarth, and M. Ch. Lux-Steiner, Phys. Rev. Lett. 97, 146601 (2006).

${ }^{18}$ D. Cahen and R. Noufi, Appl. Phys. Lett. 54, 558 (1989).

${ }^{19}$ U. Rau, D. Braunger, and H. W. Schock, Solid State Phenom. 67-68, 409 (1999).

${ }^{20}$ L. Kronik, D. Cahen, and H. W. Schock, Adv. Math. 10, 31 (1998).

${ }^{21}$ L. Kronik, U. Rau, J. F. Guillemoles, D. Braunger, H. W. Schock, and D. Cahen, Thin Solid Films 361-362, 353 (2000).

${ }^{22}$ K. Taretto, U. Rau, and J. H. Werner, Thin Solid Films 480-481, 8 (2005).

${ }^{23}$ M. Gloeckler, J. R. Sites, and W. K. Metzger, J. Appl. Phys. 98, 113704 (2005).

${ }^{24} \mathrm{~K}$. Taretto and U. Rau, in Thin-Film Compound Semiconductor Photovoltaics, edited by T. Gessert, K. Durose, C. Heske, S. Marsillac, and T. Wada, MRS Conference Proceedings No. 1012 (Materials Research Society, Pittsburgh, 2007), p. Y9-01.

${ }^{25}$ K. Orgassa, Ph.D. thesis, Universität Stuttgart, 2004.

${ }^{26}$ R. Hulstrom, R. Bird, and C. Riordan, Sol. Cells 15, 365 (1985).

${ }^{27}$ F. Engelhardt, L. Bornemann, M. Köntges, Th. Meyer, J. Parisi, E. Pschorr-Schoberer, B. Hahn, W. Gebhardt, W. Riedl, and U. Rau, Prog. Photovoltaics 7, 423 (1999).

${ }^{28}$ S. Selberherr, Analysis and Simulation of Semiconductor Devices (Springer, Vienna, 1984), p. 149.

${ }^{29}$ J. H. Werner, J. Mattheis, and U. Rau, Thin Solid Films 480-481, 399 (2005).

${ }^{30}$ S. M. Sze, Physics of Semiconductor Devices, 2nd ed. (Wiley, New York, 1981), p. 35

${ }^{31}$ For further information see: www.comsol.com.

${ }^{32}$ M. Schöfthaler, U. Rau, and J. H. Werner, J. Appl. Phys. 76, 4168 (1994).

${ }^{33}$ T. Dullweber, G. Hanna, U. Rau, and H. W. Schock, Sol. Energy Mater. Sol. Cells 67, 145 (2001).

${ }^{34}$ D. Schmid, M. Ruckh, and H. W. Schock, J. Appl. Phys. 73, 2902 (1993).

${ }^{35}$ M. Morkel, L. Weinhardt, B. Lohmüller, C. Heske, E. Umbach, W. Riedl, S. Zweigart, and F. Karg, Appl. Phys. Lett. 79, 4482 (2001).

${ }^{36}$ M. Turcu, O. Pakma, and U. Rau, Appl. Phys. Lett. 80, 2598 (2002).

${ }^{37}$ M. J. Hetzer, Y. M. Strzhemechny, M. Gao, S. Goss, M. A. Contreras, A. Zunger, and L. J. Brillson, J. Vac. Sci. Technol. B 24, 1739 (2006).

${ }^{38}$ M. J. Hetzer, Y. M. Strzhemechny, M. Gao, M. A. Contreras, A. Zunger, and L. J. Brillson, Appl. Phys. Lett. 86, 162105 (2005).

${ }^{39}$ C. Lei, C. M. Li, A. Rockett, and I. M. Robertson, J. Appl. Phys. 101, 024909 (2007).

${ }^{40}$ Y. Yan, R. Noufi, and M. M. Al-Jassim, Phys. Rev. Lett. 96, 205501 (2006).

${ }^{41}$ A. Virtuani, E. Lotter, M. Powalla, U. Rau, J. H. Werner, and M. Acciarri, J. Appl. Phys. 99, 014906 (2006).

${ }^{42}$ See, e.g., Eq. (46) on page 541 in Ref. 30.

${ }^{43}$ H. Neumann, Sol. Cells 16, 317 (1986).

${ }^{44}$ I. M. Kötschau and H. W. Schock, J. Phys. Chem. Solids 64, 1559 (2003).

${ }^{45}$ A. M. Gabor, J. R. Tuttle, D. S. Albin, M. A. Contreras, R. Noufi, and A.

M. Hermann, Appl. Phys. Lett. 65, 198 (1994).

${ }^{46}$ C. R. M. Grovenor, J. Phys. C 18, 4079 (1985). 